\title{
RIESGOS PSICOSOCIALES EN TRABAJADORES CALIFICADOS POR ENFERMEDAD LABORAL FRENTE A TRABAJADORES QUE ESPERAN POR CALIFICACIÓN
}

\author{
Nancy Vega-Gómez ${ }^{1 *}$, Harold Ceballos-Burgos ${ }^{2}$ y Julia Pardo-Carmona ${ }^{3}$ \\ ${ }^{1,2,3}$ Corporación Universitaria Unitec, Bogotá, Colombia \\ *Autor de correspondencia: 11204020@unitec.edu.co \\ Recibido Octubre 2020; Aceptado Diciembre 2020
}

\begin{abstract}
Resumen -. Los factores intrínsecos y extrínsecos a nivel organizacional junto con las enfermedades laborales generan riesgos de tipo psicosocial. El objetivo es establecer las diferencias entre los riesgos psicosociales en trabajadores calificados por enfermedad laboral frente a trabajadores que esperan por calificación. Se plantea una investigación mixta, descriptiva con alcance correlacional de los riesgos psicosociales en 10 trabajadores de una empresa de servicios generales. Los resultados cuantitativos son 3 trabajadores calificados y 7 a la espera. Los trabajadores presentan diagnósticos de 40\% Síndrome de túnel carpiano, 20\% Manguito rotador y 10\% respectivamente con Síndrome del Tendón del Tensor de la Fascia Lata, Estrés laboral, Disfonía y Lumbalgia. Los resultados cualitativos son de riesgo psicosocial medio a muy alto, concluyendo que no existen diferencias entre los riesgos psicosociales en trabajadores calificados por enfermedad laboral y trabajadores a la espera de chicha calificación, requiriendo una intervención a través de un programa de riesgos psicosociales.
\end{abstract}

Palabras clave: enfermedad laboral, riesgo psicosocial, factor psicosocial, nivel de riesgo psicosocial, estrés.

\section{PSYCHOSOCIAL RISKS IN WORKERS QUALIFIED BY OCCUPATIONAL ILLNESS VS. WORKERS WHO WAIT FOR QUALIFICATION}

\begin{abstract}
Intrinsic and extrinsic factors at the organizational level together with occupational diseases generate a psychosocial risk. The objective is to establish the differences between the psychosocial risks in workers qualified by occupational disease versus workers waiting for qualification. A mixed, descriptive investigation with correlational scope of psychosocial risks in 10 workers of a general services company is proposed. The quantitative results are 3 skilled workers and 7 waiting. The workers present diagnoses of 40\% Carpal Tunnel Syndrome, 20\% Rotator Cuff and 10\% respectively with Tendon Fascia Lata Tendon Syndrome, Work Stress, Dysphonia and Low Back Pain. The qualitative results are of medium to extremely high psychosocial risk, concluding that there are no differences between the psychosocial risks in workers qualified by occupational disease and workers awaiting qualification, requiring an intervention through a program of psychosocial risks.
\end{abstract}

Keywords: occupational disease, psychosocial risk, psychosocial factor, level of psychosocial risk, stress. 
Riesgos psicosociales en trabajadores calificados por enfermedad laboral frente a trabajadores que esperan por calificación

\section{Introducción}

El hombre como ser social, demanda la actividad productiva, siendo primordial para su progreso físico, económico, social, espiritual y psicológico, pero de igual forma, estos propios mecanismos pueden ser los que suscitan alguna variabilidad en la salud de los individuos (González, 2016, p. 817), trayendo como consecuencia un deterioro o pérdida de la integralidad a nivel personal, social, de salud, administrativo, laboral y económico.

Las organizaciones a nivel nacional, sin distinguir su actividad económica, tamaño, forma jurídica y origen de capital (Ministerio del Trabajo, 2014, p. 2), deben establecer un Sistema de Gestión de la Seguridad y Salud en el Trabajo (SG-SST) en el cual se incluya la "identificación y evaluación de los factores psicosociales propios del trabajo, así como sus efectos; alcanzando aspectos internos y externos de cualquier empresa, donde deben ser incluidas las características específicos de los trabajadores, cuyos conocimientos y experiencias, influyen de manera activa en la salud y en el desarrollo de un empleado en cualquier organización" (Ministerio de la Protección Social, 2008, p. 3).

Los riesgos psicosociales, menciona el organismo UGT Andalucía (UGT Andalucía, 2009, p. 7), a pesar de "ser uno de los factores que poseen mayor incidencia en la cotidianidad de las organizaciones, no tienen el suficiente estudio para ser identificados y controlados", eliminados o minimizados, por lo tanto, requieren de una efectiva planificación de actividades de intervención de acuerdo con la identificación y valuación del riesgo.

Por tal motivo, visto este enfoque desde la correlación existente entre la condición laboral y la salud del trabajador se puede inferir que algunas condiciones de trabajo son elementos que interceden de forma perceptible en la salud (Cacua et al, 2017, p. 101), por ende, cualquier cambio en dichas condiciones dará lugar a situaciones de alteración en la salud integral del colaborador.

Por consiguiente, se realiza un abordaje de la temática de riesgos psicosociales en trabajadores que hayan alcanzado la calificación de pérdida de capacidad laboral con origen en una enfermedad laboral frente a trabajadores que se encuentren en proceso de dicha calificación de enfermedad, lo que permite la producción de un texto científico con el objetivo de transferir el resultado obtenido (Ruiz \& López, 2020, p. 140), optimizando la toma de decisiones tanto para los especialistas en SST como para las mismas empresas.

Este abordaje, se hace a través de la aplicación de la batería de riesgo psicosocial, establecida por la Universidad Javeriana junto con el Ministerio de la Protección social según lineamientos establecidos en la Resolución 2646 de 2008 (Ministerio de la Protección Social, 2008, p. 2), instrumento que permite identificar si los trabajadores se encuentran bajo estresores de riesgo psicosocial.

De igual forma, el presente trabajo busca establecer la correlación en trabajadores que esperan la calificación de pérdida de capacidad laboral por enfermedad laboral debido a la presencia de cuadros de riesgos psicosociales adicionales a las condiciones físicas de salud propias de la 
enfermedad, o si los trabajadores que ya obtuvieron una respuesta de calificación de la enfermedad laboral desarrollan poco o nada la aparición de los riesgos psicosociales.

Para finalizar, los resultados obtenidos permitirán realizar un abordaje de recomendaciones de intervención y control, no sólo para los trabajadores que se encuentren en el proceso de calificación de enfermedad laboral, sino que incluyan a la población trabajadora de toda la organización, permitiendo optimizar las condiciones de la vida laboral y aumentando el nivel de confianza en todos los actores del sistema empresarial (Riaño, 2017, p. 29).

\section{Materiales y Métodos}

En este proceso investigativo se maneja un tipo de estudio mixto, es decir, metodologías que utilizan evidencia de datos numéricos, verbales, textuales, y de otra índole para comprender el problema presentado, lo que implica un trabajo multidisciplinario, a través de la conformación de grupos de personas con incentivos variados pero que convergen en un propósito común, y es el de dar respuesta al planteamiento de la pregunta de investigación. (Hernández et al, 2014, p. 129).

El diseño de la investigación es de tipo descriptivo, ya que se realiza la "caracterización de un hecho, fenómeno, individuo o grupo, con el fin de establecer su estructura y comportamiento" (Arias, 2012, p. 111).

De igual forma, la investigación tiene un alcance correlacional, pues su finalidad es determinar el grado de relación o asociación existente entre dos o más variables, en donde primero se miden las variables y luego mediante la aplicación de técnicas estadísticas de análisis inferencial se comparan los resultados (Flores et al, 2017, p. 365) entre los trabajadores calificados con los que esperan calificación de enfermedad de origen laboral, estimando la correlación, lo que aporta indicios sobre las posibles causas de un fenómeno (Arias, 2012, p. 26), que en este caso son los riesgos psicosociales respecto a la calificación o espera de calificación de pérdida de capacidad laboral.

De igual forma a través del análisis inferencial se logra establecer la relación existente entre la pregunta de investigación, la hipótesis y los objetivos lo que permite correlacionar las variables, detectando los factores que mayor influencia tienen en la población objeto de estudio (Ramos, 2019, p. 9).

En el presente trabajo de investigación se tienen en cuenta datos obtenidos en primera instancia de documentos o artículos publicados en revistas científicas de bases de datos como PubMed, REDIB, Latindex, Redalyc, Scielo, Dialnet, LILACS, y documentos de la administración pública colombiana a través del Diario Oficial, divulgaciones de entes gubernamentales.

La batería de instrumentos para la evaluación de factores de riesgo psicosocial es aplicada en una empresa de servicios generales de la ciudad de Cartagena de Indias; Colombia, a un número de 10 trabajadores, de los cuales 3 ya presentan calificación de pérdida de capacidad laboral por origen laboral y 7 que se encuentran en proceso de calificación de enfermedad laboral. 
Son criterios de exclusión para el estudio, trabajadores que no pertenezcan a la empresa de servicio generales contactada y que no presenten calificación de enfermedad laboral o que no estén en proceso de trámite de su calificación por enfermedad laboral.

Para el desarrollo de este ejercicio se emplean 5 cuestionarios de la batería de evaluación de riesgo psicosocial proporcionada por el Ministerio de la Protección Social y la Universidad Javeriana. Los 5 instrumentos aplicados son: el consentimiento informado, la ficha de datos generales, el cuestionario para la evaluación del estrés, el cuestionario de factores psicosociales extralaborales y el cuestionario de factores de riesgo psicosocial intralaboral en su forma B, ya que ninguno de los trabajadores encuestados es jefe de área.

Los resultados de las baterías aplicadas deben ser tabulados y correlacionados en Microsoft Excel y en el paquete estadístico RStudio. Microsoft Excel, funciona como programa informático que permite realizar cálculos de datos y obtener un sin número de posibilidades de correlación de estos, mostrándose en forma de tabla o de gráfica, donde las hojas de cálculo u hoja electrónica admiten ejecutar procesamiento de datos sencillos y complejos con rapidez y precisión, accediendo a crear o simular escenarios con el objetivo de desarrollar análisis sobre las mismos.

Por otra parte, RStudio versión 4.0.2, es un conjunto de programas integrados para el manejo de datos, simulaciones, cálculos y realización de gráficos. Es un lenguaje apropiado para la estadística, ya que permite manipular los datos rápidamente y de forma precisa. R como lenguaje orientado a objetos, permite el tratamiento de la información a nivel estadístico y la creación de gráficos, ofreciendo una gran diversidad de metodologías estadísticas y gráficas, gracias al anexo de nuevas técnicas mediante la definición de funciones.

Como resultado se busca que todos las etapas de este proceso de investigación sean utilizados desde lo particular hasta llegar a las conclusiones generales (Andrade et al, 2018, p. 118), que en conjunto con las bases conceptuales permite comprender la forma de integrar los datos y sus resultados a través de métodos estadísticos inferenciales (Moreno, 2018, p. 759), con la única finalidad de entender el fenómeno de los riesgos psicosociales tanto en trabajadores calificados por enfermedad laboral como por los empleados que se encuentran a la espera de dicha calificación.

\section{Resultados y Discusión}

A continuación, se hace la presentación de los principales resultados obtenidos de la aplicación de los instrumentos de fichas de datos generales y la batería de factores psicosociales.

En el desarrollo de los resultados, los primeros corresponden al porcentaje de estado de calificados o a la espera de calificación de enfermedad de origen laboral, en segunda instancia los resultados pertenecen a los porcentajes en cuanto a los diagnósticos de enfermedad laboral calificada y en estudio que los trabajadores poseen. Por último, se presentan las correlaciones correspondientes entre los factores intralaborales, extralaborales y estrés con sus correspondientes dominios y variables de cada uno de estos. Para este último análisis se realiza 

trabajadores que esperan por calificación

en tres tiempos, 1) correlación general, 2) correlación entre trabajadores calificados y 3) correlación entre trabajadores a la espera de calificación.

A continuación, se presentan los siguientes resultados: en la Figura 1, el porcentaje de trabajadores que ya poseen una calificación de enfermedad de origen laboral es de $30 \%$ y el porcentaje de trabajadores que se encuentran en proceso de calificación de enfermedad laboral es de $70 \%$. En la figura 2, se encuentran los porcentajes de diagnóstico de enfermedad laboral de los trabadores Síndrome de Túnel Carpiano (40\%), Hombro Doloroso (20\%) y Lumbalgia, Disfonía, Estrés Laboral (10\%) y Síndrome del Tendón de la Fascia Lata; cada uno con 10\% respectivamente.

Figura 1: Calificación

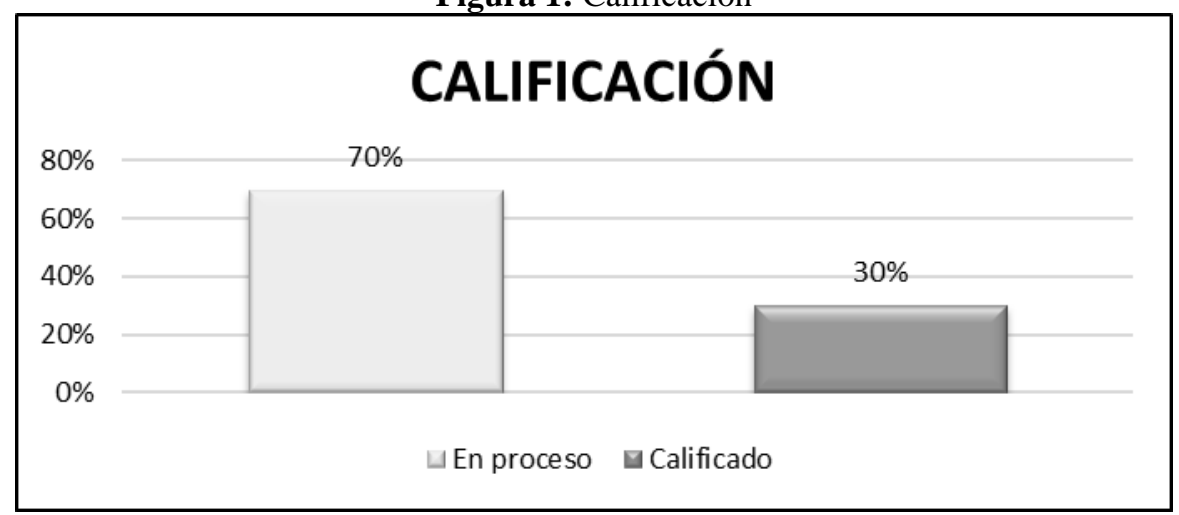

Fuente: Elaboración propia

Figura 2: Diagnóstico

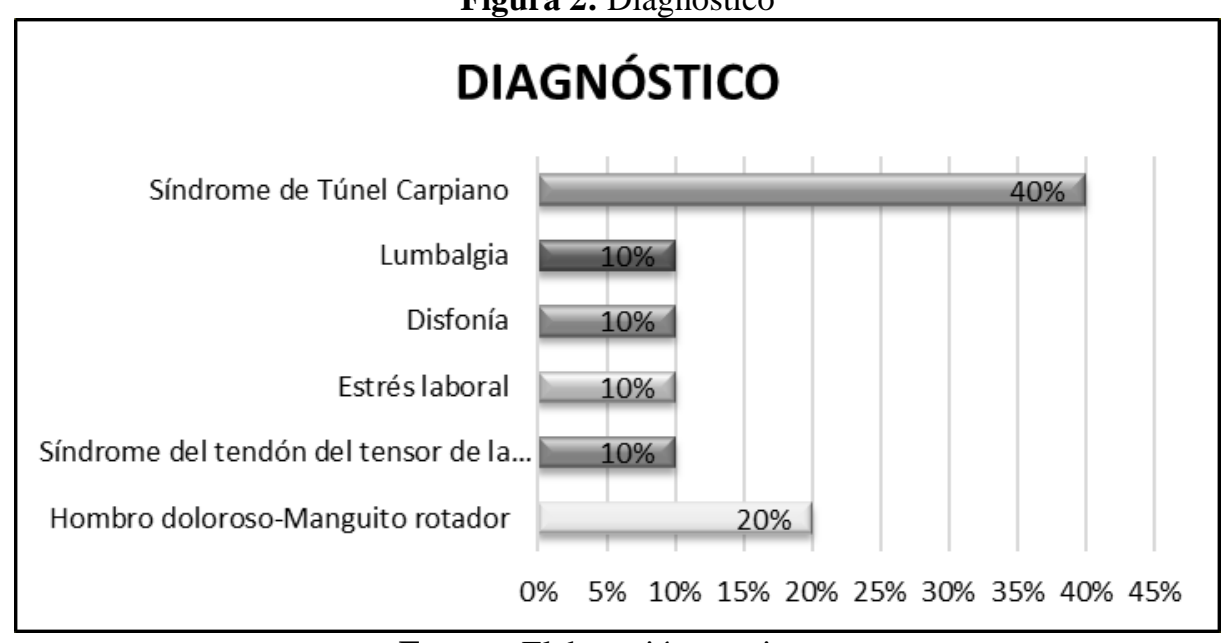

Fuente: Elaboración propia

Los valores aportados en la Tabla 1, son los correspondientes a los niveles de riesgo obtenidos de la aplicación de las baterías de riesgo psicosocial de acuerdo con los factores laborales tanto Intrínsecos como Extrínsecos y el nivel de riesgo para el Estrés Laboral, con sus correspondientes dominios. 
Riesgos psicosociales en trabajadores calificados por enfermedad laboral frente a trabajadores que esperan por calificación

Tabla 1: Valores totales nivel de riesgo batería riesgo psicosocial

\begin{tabular}{|c|c|c|c|c|c|c|}
\hline \multicolumn{2}{|c|}{ INTRALABORALES } & $\begin{array}{l}\text { Sin Riesgo - } \\
\text { Despreciable }\end{array}$ & Bajo & Medio & Alto & $\begin{array}{l}\text { Muy } \\
\text { Alto }\end{array}$ \\
\hline \multirow{3}{*}{$\begin{array}{l}\text { Liderazgo y } \\
\text { relaciones } \\
\text { sociales en el } \\
\text { trabajo }\end{array}$} & Características del liderazgo & $0 \%$ & $10 \%$ & $20 \%$ & $30 \%$ & $40 \%$ \\
\hline & Relaciones sociales en el trabajo & $0 \%$ & $0 \%$ & $0 \%$ & $0 \%$ & $100 \%$ \\
\hline & Retroalimentación del desempeño & $0 \%$ & $20 \%$ & $10 \%$ & $50 \%$ & $20 \%$ \\
\hline \multirow{5}{*}{$\begin{array}{l}\text { Control sobre } \\
\text { el trabajo }\end{array}$} & Claridad de rol & $10 \%$ & $30 \%$ & $0 \%$ & $10 \%$ & $50 \%$ \\
\hline & Capacitación & $10 \%$ & $10 \%$ & $30 \%$ & $20 \%$ & $30 \%$ \\
\hline & $\begin{array}{c}\text { Participación y manejo del } \\
\text { cambio }\end{array}$ & $0 \%$ & $10 \%$ & $30 \%$ & $40 \%$ & $20 \%$ \\
\hline & $\begin{array}{c}\text { Oportunidades para el uso y } \\
\text { desarrollo de habilidades y } \\
\text { conocimientos }\end{array}$ & $20 \%$ & $10 \%$ & $0 \%$ & $50 \%$ & $20 \%$ \\
\hline & $\begin{array}{l}\text { Control y autonomía sobre el } \\
\text { trabajo }\end{array}$ & $30 \%$ & $0 \%$ & $20 \%$ & $10 \%$ & $40 \%$ \\
\hline \multirow{6}{*}{$\begin{array}{l}\text { Demandas } \\
\text { del trabajo }\end{array}$} & $\begin{array}{c}\text { Demandas ambientales y de } \\
\text { esfuerzo físico }\end{array}$ & $0 \%$ & $10 \%$ & $20 \%$ & $30 \%$ & $40 \%$ \\
\hline & Demandas emocionales & $40 \%$ & $10 \%$ & $40 \%$ & $0 \%$ & $10 \%$ \\
\hline & Demandas cuantitativas & $0 \%$ & $10 \%$ & $10 \%$ & $10 \%$ & $70 \%$ \\
\hline & $\begin{array}{c}\text { Influencia del trabajo sobre el } \\
\text { entorno extralaboral }\end{array}$ & $0 \%$ & $0 \%$ & $0 \%$ & $60 \%$ & $40 \%$ \\
\hline & Demandas de carga mental & $10 \%$ & $20 \%$ & $10 \%$ & $30 \%$ & $30 \%$ \\
\hline & $\begin{array}{c}\text { Demandas de la jornada de } \\
\text { trabajo }\end{array}$ & $10 \%$ & $30 \%$ & $20 \%$ & $10 \%$ & $30 \%$ \\
\hline \multirow[t]{2}{*}{ Recompensas } & $\begin{array}{l}\text { Recompensas derivadas de la } \\
\text { pertenencia a la organización y } \\
\text { del trabajo que se realiza }\end{array}$ & $20 \%$ & $10 \%$ & $0 \%$ & $0 \%$ & $70 \%$ \\
\hline & Reconocimiento y compensación & $10 \%$ & $0 \%$ & $10 \%$ & $20 \%$ & $60 \%$ \\
\hline \multicolumn{2}{|c|}{ EXTRALABORALES } & $\begin{array}{l}\text { Sin Riesgo - } \\
\text { Despreciable }\end{array}$ & Bajo & Medio & Alto & $\begin{array}{l}\text { Muy } \\
\text { Alto }\end{array}$ \\
\hline \multicolumn{2}{|c|}{ Tiempo fuera del trabajo } & $0 \%$ & $10 \%$ & $20 \%$ & $20 \%$ & $50 \%$ \\
\hline \multicolumn{2}{|r|}{ Relaciones Familiares } & $0 \%$ & $80 \%$ & $0 \%$ & $10 \%$ & $10 \%$ \\
\hline \multicolumn{2}{|c|}{ Comunicación y relaciones interpersonales } & $10 \%$ & $0 \%$ & $30 \%$ & $20 \%$ & $40 \%$ \\
\hline \multicolumn{2}{|c|}{ Situación económica del grupo familiar } & $30 \%$ & $0 \%$ & $20 \%$ & $0 \%$ & $50 \%$ \\
\hline \multicolumn{2}{|c|}{ Características de la vivienda y de su entorno } & $0 \%$ & $50 \%$ & $0 \%$ & $20 \%$ & $30 \%$ \\
\hline \multicolumn{2}{|c|}{ Influencia del entorno extralaboral sobre el trabajo } & $0 \%$ & $20 \%$ & $30 \%$ & $20 \%$ & $30 \%$ \\
\hline \multicolumn{2}{|c|}{ Desplazamiento vivienda - trabajo - vivienda } & $0 \%$ & $20 \%$ & $30 \%$ & $10 \%$ & $40 \%$ \\
\hline \multirow{2}{*}{\multicolumn{2}{|c|}{ ESTRÉS }} & $\begin{array}{l}\text { Sin Riesgo - } \\
\text { Despreciable }\end{array}$ & Bajo & Medio & Alto & $\begin{array}{l}\text { Muy } \\
\text { Alto }\end{array}$ \\
\hline & & $0 \%$ & $0 \%$ & $20 \%$ & $0 \%$ & $80 \%$ \\
\hline
\end{tabular}

Fuente: Elaboración propia

Las cifras aportadas por la Tabla 2, son las correspondientes a la correlación general tanto positiva como negativa entre los factores intrínsecos, factores extrínsecos y estrés laboral, del grupo de trabajadores objeto de estudio; es decir, trabajadores calificados con enfermedad de origen laboral y trabajadores a la espera de dicha calificación. 
Riesgos psicosociales en trabajadores calificados por enfermedad laboral frente a trabajadores que esperan por calificación

Tabla 2: Correlación general

\begin{tabular}{ccccccc}
\hline GENERAL & $\begin{array}{c}\text { Liderazgo y } \\
\text { relaciones sociales } \\
\text { en el trabajo }\end{array}$ & $\begin{array}{c}\text { Control } \\
\text { sobre el } \\
\text { trabajo }\end{array}$ & $\begin{array}{c}\text { Demandas } \\
\text { del trabajo }\end{array}$ & Recompensas & $\begin{array}{c}\text { Tiempo } \\
\text { fuera del } \\
\text { trabajo }\end{array}$ & ESTRÉS \\
\hline $\begin{array}{c}\text { Liderazgo y } \\
\text { relaciones sociales } \\
\text { en el trabajo }\end{array}$ & 1 & & & & \\
$\begin{array}{c}\text { Control sobre el } \\
\text { trabajo }\end{array}$ & 0,927190 & 1 & & & \\
$\begin{array}{c}\text { Demandas del } \\
\text { trabajo } \\
\text { Recompensas }\end{array}$ & 0,821724 & 0,699555 & 1 & 1 & \\
$\begin{array}{c}\text { Tiempo fuera del } \\
\text { trabajo }\end{array}$ & 0,153702 & 0,246554 & $-0,001799$ & & \\
ESTRÉS & 0,497276 & 0,439187 & 0,334464 & $-0,287026$ & 1 & \\
\hline
\end{tabular}

Fuente: Elaboración propia

En la Figura 3, se presenta de manera gráfica y numérica la correspondiente correlación entre los factores intrínsecos, factores extrínsecos y estrés laboral de los trabajadores objeto de estudio, dando como resultado una correlación positiva, de forma que, si el dominio de liderazgo y relaciones sociales es mayor, también es mayor el dominio de control sobre el trabajo, con una relación fuerte de 0.927190 . Mientras que la correlación negativa, se presenta cuando a mayor dominio del tiempo fuera del trabajo, menor es el dominio de recompensa, siendo esta relación débil con un valor de -0.287026 .

Figura 3: Correlación general

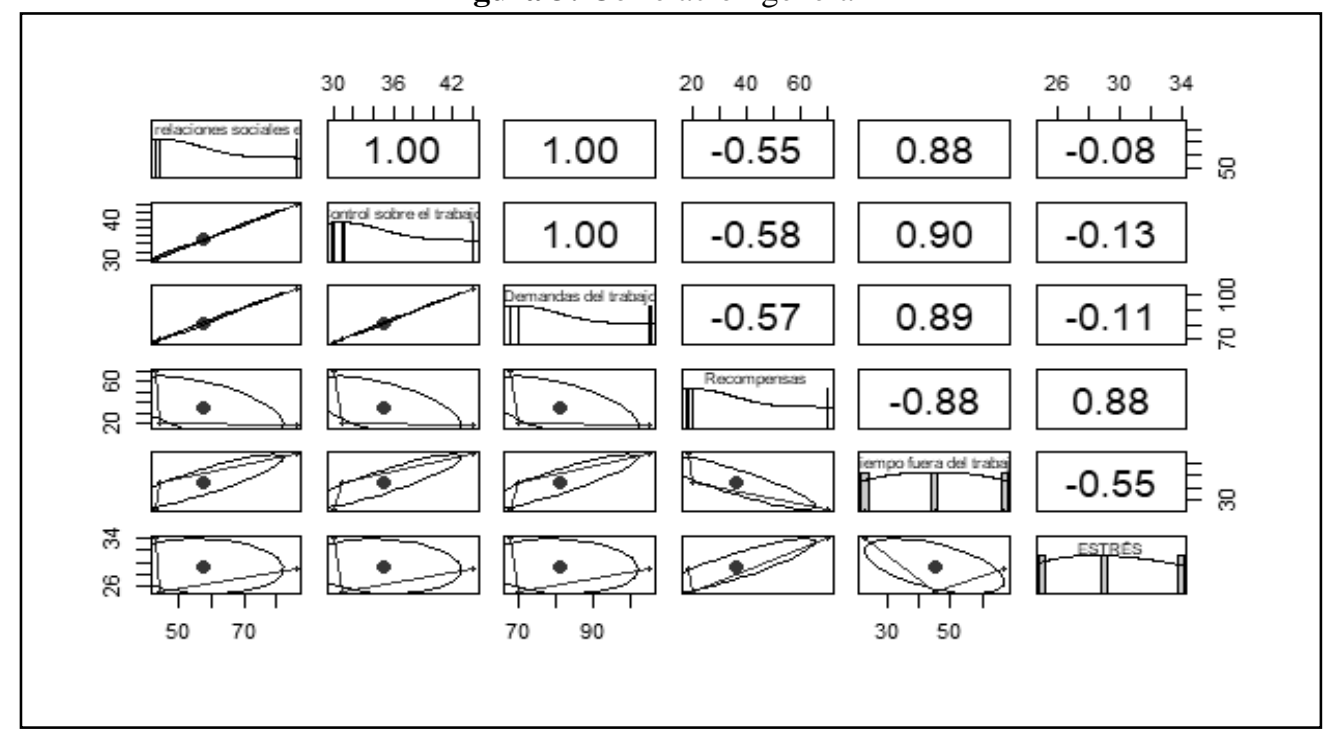

Fuente: Elaboración propia

En la Tabla 3, se encuentran los datos correspondientes las cifras de correlaciones tanto positivas como negativas de los factores intrínsecos, factores extrínsecos y estrés laboral, específicamente para los trabajadores que poseen calificación de enfermedad laboral. 
Riesgos psicosociales en trabajadores calificados por enfermedad laboral frente a trabajadores que esperan por calificación

Tabla 3: Correlación en trabajadores calificados

\begin{tabular}{ccccccc}
\hline $\begin{array}{c}\text { Trabajadores } \\
\text { Con } \\
\text { Calificación }\end{array}$ & $\begin{array}{c}\text { Liderazgo y } \\
\text { relaciones } \\
\text { sociales en } \\
\text { el trabajo }\end{array}$ & $\begin{array}{c}\text { Control } \\
\text { sobre el } \\
\text { trabajo }\end{array}$ & $\begin{array}{c}\text { Demandas } \\
\text { del trabajo }\end{array}$ & $\begin{array}{c}\text { Recompensa } \\
\mathrm{s}\end{array}$ & $\begin{array}{c}\text { Tiempo } \\
\text { fuera del } \\
\text { trabajo }\end{array}$ & ESTRÉS \\
\hline $\begin{array}{c}\text { Liderazgo y } \\
\text { relaciones } \\
\text { sociales en el } \\
\text { trabajo }\end{array}$ & 1 & & & & & \\
$\begin{array}{c}\text { Control sobre } \\
\text { el trabajo }\end{array}$ & 0,999046 & 1 & & & & \\
$\begin{array}{c}\text { Demandas } \\
\text { del trabajo }\end{array}$ & 0,999616 & 0,999872 & 1 & & & \\
$\begin{array}{c}\text { Recompensas } \\
\text { Tiempo fuera } \\
\text { del trabajo }\end{array}$ & $-0,546284$ & $-0,582345$ & $-0,569274$ & 1 & & \\
ESTRÉS & $-0,0846032$ & 0,896258 & 0,889053 & $-0,882498$ & 1 & \\
\hline
\end{tabular}

Fuente: Elaboración propia

En la figura 4, se evidencia la correlación de los trabajadores calificados tanto numérica como gráficamente, de acuerdo con los datos de nivel de riesgo en los factores intrínsecos, factores extrínsecos y estrés laboral. Según los datos aportados, en el caso de los trabajadores que ya poseen definición de calificación por enfermedad de origen laboral, se presenta una correlación positiva, ya que entre mayor es el dominio control sobre el trabajo, mayor es el dominio demanda del trabajo, siendo esta relación fuerte con un valor de 0.999872. Mientras que se da una correlación negativa, cuando entre mayor es el tiempo fuera del trabajo, menor es el dominio de la recompensa, cuyo valor es de -0.882498; siendo esta una relación fuerte también.

Figura 4: Correlación en trabajadores calificados

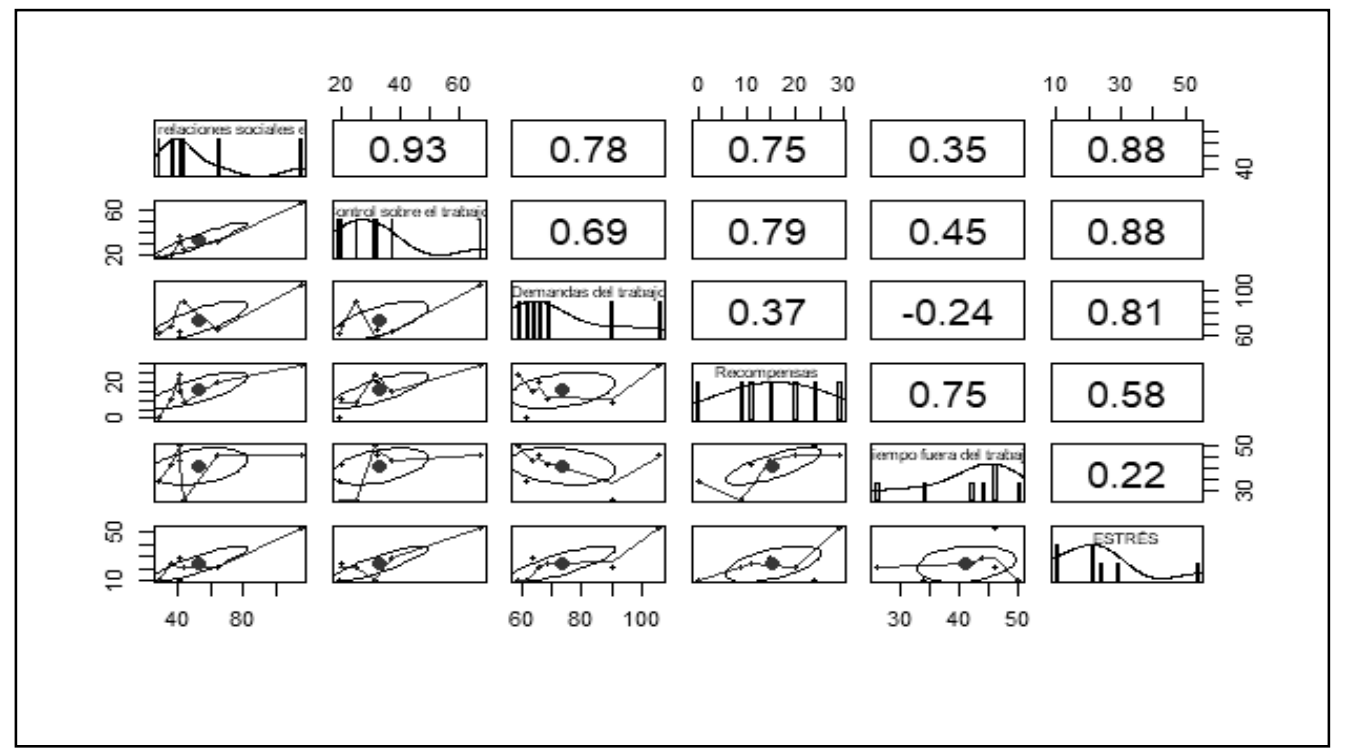

Fuente: Elaboración propia 
Respecto a la Tabla 4, se muestra las correlaciones tanto positivas como negativas de los niveles de riesgo para los factores intrínsecos, factores extrínsecos y estrés laboral, pero exclusivamente para los trabajadores que esperan por calificación de enfermedad laboral.

Tabla 4: Correlación en trabajadores por calificación

\begin{tabular}{|c|c|c|c|c|c|c|}
\hline $\begin{array}{c}\text { Trabajadores a la } \\
\text { espera de } \\
\text { Calificación }\end{array}$ & $\begin{array}{l}\text { Liderazgo y } \\
\text { relaciones } \\
\text { sociales en } \\
\text { el trabajo }\end{array}$ & $\begin{array}{l}\text { Control } \\
\text { sobre el } \\
\text { trabajo }\end{array}$ & $\begin{array}{c}\text { Demandas } \\
\text { del } \\
\text { trabajo }\end{array}$ & Recompensas & $\begin{array}{l}\text { Tiempo } \\
\text { fuera del } \\
\text { trabajo }\end{array}$ & ESTRÉS \\
\hline $\begin{array}{l}\text { Liderazgo y } \\
\text { relaciones } \\
\text { sociales en el } \\
\text { trabajo }\end{array}$ & 1 & & & & & \\
\hline $\begin{array}{c}\text { Control sobre el } \\
\text { trabajo }\end{array}$ & 0,934650 & 1 & & & & \\
\hline $\begin{array}{l}\text { Demandas del } \\
\text { trabajo }\end{array}$ & 0,779862 & 0,693320 & 1 & & & \\
\hline Recompensas & 0,750893 & 0,792128 & 0,366746 & 1 & & \\
\hline $\begin{array}{l}\text { Tiempo fuera del } \\
\text { trabajo }\end{array}$ & 0,352295 & 0,445943 & $-0,235619$ & 0,748777 & 1 & \\
\hline ESTRÉS & 0,875797 & 0,881731 & 0,807277 & 0,576855 & 0,224222 & 1 \\
\hline
\end{tabular}

Fuente: Elaboración propia

Figura 5: Correlación en trabajadores por calificación

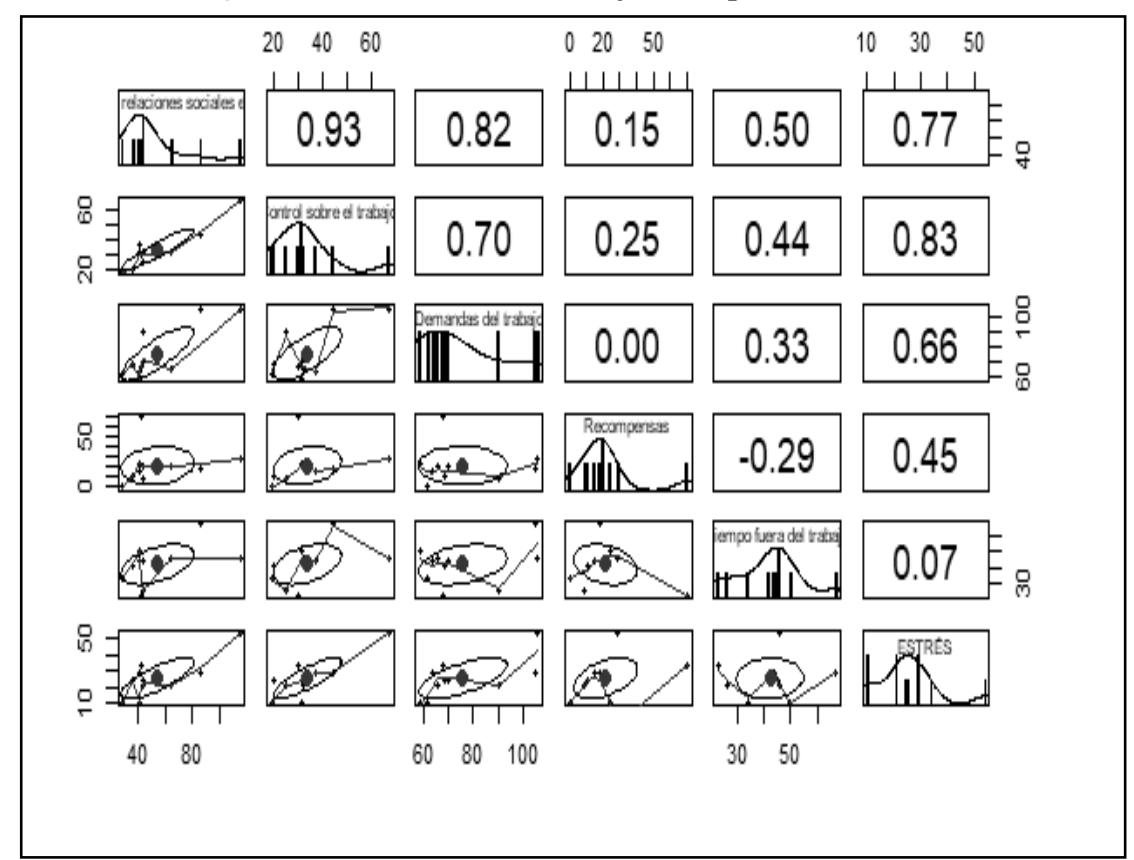

Fuente: Elaboración propia

Por último, se observa en la Figura 5, los datos numéricos y gráficos de las correlaciones de los niveles de riesgo de los factores intrínsecos, factores extrínsecos y estrés laboral, para los 
trabajadores que se encuentran a la espera de calificación por enfermedad laboral. Las correlaciones en este grupo son positivas cuando a mayor dominio del liderazgo y las relaciones sociales en el trabajo, mayor es el dominio control sobre el trabajo. Pero la correlación es negativa cuando se presenta mayor dominio de tiempo fuera del trabajo, menor es el dominio de demandas en el trabajo. La correlación directa tiene relación fuerte con un valor de 0.934650 y la correlación indirecta tiene un valor de -0.235618, siendo esta débil.

Por este motivo, se aborda la Higiene Industrial, como una ciencia encargada de identificar, evaluar y controlar los riesgos cuyo origen se encuentra en los entornos de trabajo y que pueden en cierta medida repercutir en la salud de sus colaboradores, obligando a la alta gerencia a tomar las medidas tanto correctivas como preventivas en materia de riesgos laborales. En el caso de la empresa de servicios generales, aunque dichos riesgos incluyendo los psicosociales, ya se encuentre identificados y evaluados, aún estos no han sido tratados, ni controlados. Se revela este escenario en los colaboradores objeto de estudio, debido a que los sujetos exponen niveles elevados de estrés laboral, es decir, no han percibido un acompañamiento en el desarrollo de su enfermedad, ni en su reincorporación a una vida social y laboral que cumpla con estándares los estándares mínimos de calidad para su vida.

Es aquí donde intervienen con un papel esencial los factores de riesgo psicosociales, con el objetivo de precisar cuáles son las circunstancias de trabajo existentes en los ambientes laborales y el modo en que estos inciden de manera positiva y negativa en la salud del trabajador (Organización Internacional del Trabajo, 2020, p. 1). Por tal motivo, se hace necesario establecer dichas condiciones laborales y su capacidad de afectación en cada uno de los individuos que pertenecen a la organización (UGT Andalucía, 2009, p. 18), haciéndose primordial el establecimiento en la identificación de los riesgos que se generan a nivel ocupacional, los cuales deben ser intervenidos de manera pertinente en tiempo, lugar e individuo, evitando la afectación de la salud de los empleados (Herrán et al, 2016, p. 44), así como en la continuidad del negocio.

Una de las actividades de acompañamiento, se refiere a la reubicación laboral, que a pesar de estar ya establecida para los 3 trabajadores calificados, las modificaciones en su estilo de vida, los aspectos laborales, familiares y su entorno (Delgado \& Aguilera, 2017, p. 21), no ha sido pertinente al momento de mejorar su calidad de vida, por el contrario, sus niveles de riesgo son altos o muy altos, lo que a posterioridad puede exponer un horizonte nada alentador para los colaboradores que se encuentran a la espera de calificación. Dicha situación inclusive puede traer manifestaciones de distrés elevado o muy elevado con sintomatología depresiva como lo manifiesta la autora Ansoleaga (Ansoleaga, 2015, p. 53).

Por otra parte, según lo que refiere la autora Elda Gonzáles, respecto a una correlación baja entre el estrés laboral y los riesgos psicosociales (Gonzales, 2017, p. 64), no aplica a este estudio, porque como se evidencia en las correlaciones a nivel de estrés todas obtienen una calificación entre media y muy alta, con una correlación fuerte especialmente entre estrés y riesgos intralaborales (recompensas).

Esta variación puede darse de manera diferente en las organizaciones, ya que la forma de abordar la identificación y el manejo de los riesgos psicosociales y el estrés, debe siempre estar bajo lineamientos de planes de mejora continua y seguimiento a los trabajadores que requieran mayor IPSA SCIENTIA - Vol. 5 No $1-2020$ 
atención, todo enmarcado en la implementación de programas de riesgo psicosocial tal y como lo mencionan Lars Moreno y colaboradores (Moreno et al., 2018, p. 164), situación que no se ve reflejada en los resultados de la aplicación de los cuestionarios de riesgos psicosociales en la población objeto de estudio.

También es preciso tener en cuenta que este o cualquier otro colectivo de colaboradores que se encuentre frente a la presencia o investigación de una enfermedad de origen laboral, debe apartarse de su trabajo en múltiples ocasiones debido a la asistencia a los servicios médicos que se les brinda para el cuidado y seguimiento de su patología a nivel físico y mental, según lo plantean los autores Magally Otárola (Otárola, 2015, p. 77) y Patricia Arias y colaboradores (Arias et al, 2012, p. 18), en especial evitando que dichas situaciones sigan incrementando el nivel de riesgo en cuanto a estrés y factores de riesgo psicosocial.

De igual forma, Lorena Garzón (Garzón, 2019, p. 9), enfatiza que tanto las restricciones como las recomendaciones médicas ocupacionales deben siempre estar encaminadas a proteger la salud integral de los trabajadores en general y especialmente en colaboradores que tengan o estén a la espera de la calificación de enfermedad de origen laboral. Se debe por lo tanto incluir dentro del proceso, el apoyo emocional brindando un trato y trabajo digno de acuerdo con sus capacidades actuales.

Por tal motivo, todo modelo de aplicación frente al tratamiento de los riesgos psicosociales debe definir elementos internos y externos del trabajo que, sumados a los contextos individuales del trabajador, permitan establecer las interrelaciones dinámicas, por medio de percepciones y experiencias, que contribuyan en el bienestar y la competencia de las personas (Rubio \& Luna, 2014, p. 35).

Por ende, es importante que el mantenimiento de los recursos para el desarrollo de todas las actividades y programas encaminados a la reubicación laboral, así como al tratamiento de los factores de riesgo preponderantes en la organización, se den siempre de manera ininterrumpida.

Aunque en el caso de los trabajadores objeto de estudio no se tiene precisión sobre el seguimiento y el acompañamiento de los trabajadores en su proceso referente a la enfermedad laboral, los resultados obtenidos con la aplicación de la batería de riesgo psicosocial, muestra un panorama de no control y seguimiento a estos empleados, reflejados en sus niveles altos de estrés y riesgo psicosocial.

De igual forma, también es importante reconocer que los escenarios del trabajo no son los únicos que causan estas condiciones. Las situaciones estresantes también se dan fuera del trabajo, incluyendo los entornos domésticos, crean barreras en las personas, los cuales se expresan en un estado de ánimo afectado (Gómez \& Calderón, 2017, p. 180).

Es importante destacar que las baterías de evaluación de riesgos psicosociales son herramientas útiles a la hora de identificar dichos factores de riesgo, ya que abordan diversas variables tanto intralaborales como extralaborales, según los planteamientos establecidos por Viviola Gómez y colaboradores (Gómez et al, 2016, p. 225), donde lo importante es obtener las calificaciones que permitan conseguir diagnósticos confiables según lo estipulado por la Resolución 2646:2008, para determinar los programas a implementar en la organización según resultados tanto a nivel IPSA SCIENTIA - Vol. 5 No $1-2020$ 
individual como grupal, así como los mínimos obligatorios para identificación, evaluación, monitoreo e intervención de los riesgos psicosociales según la Resolución 2404:2019. En la organización a la que pertenecen los trabajadores objeto de estudio, solo han logrado la identificación y evaluación de dichos riesgos psicosociales, pero les falta continuar con el ciclo de implementación y mejora de los programas referentes a factores psicosociales.

Es clave destacar la medida de protección que desde la Corte Constitucional de Colombia se ha dado para los trabajadores que se encuentren en proceso o que ya posean una calificación de enfermedad por origen laboral, tal y como lo presentan los autores Mauricio Torres y Nicolas Torres (Torres \& Torres, 2017, p. 776), donde a pesar de no tener una respuesta positiva en primera instancia, es la Corte a través de sentencias que ratifica el derecho a la reubicación laboral y sus correspondientes prestaciones asistenciales y económicas de los trabajadores a la espera o que ya poseen una calificación de enfermedad laboral, sin que esta situación les genere incertidumbres respecto a su situación laboral futura.

La reubicación laboral se encuentra amparada jurídicamente en Colombia (Congreso de Colombia, 2002, p. 4), a través de la obligatoriedad que se establece para que los empleadores proporcionen trabajos dignos y compatibles con la capacidad y aptitudes de dichos empleados, situación que se ve reflejada en la organización con la reubicación de los 3 trabajadores que ya poseen una calificación de enfermedad por origen laboral. Por otra parte, es necesario mencionar que los trabajadores que se encuentran a la espera de calificación por enfermedad laboral no han sido desamparados, ellos siguen haciendo parte de la organización a través de contrato de trabajo.

De igual forma es importante establecer que lograr un clima laboral se presenta como una de las características primordiales de cualquier organización, en donde cada uno de los miembros que la integran buscan que sus sentimientos y representaciones (mentales, sociales y cognitivas) de los elementos que la conforman y de la forma como se instauran y dan cumplimiento de estas como son las políticas, procedimientos de trabajo, estilos de liderazgo, funciones, deberes y responsabilidades, que permiten alcanzar una organización eficaz y eficiente tanto a nivel personal como a nivel empresarial (Muñoz et al, 2018, p. 18).

De todo lo anteriormente expuesto, surge la necesidad de formar líderes que se involucren con las necesidades de sus trabajadores, tal y como lo plantea Fanny Gómez (Gómez, 2019, p. 13), debido a que si no se le proporciona al trabajador estímulos y apoyo emocional pueden incrementarse los casos de altos niveles de riesgos psicosociales, tal y como sucede en los trabajadores participantes del estudio, en donde el programa de riesgo psicosocial no ha sido llevado a cabalidad siendo una de las causas posibles la falta de liderazgo en el proceso, lo que no permite reconocer los esfuerzos y resultados conseguidos por parte de los trabajadores.

\section{Conclusiones}

De acuerdo con los resultados obtenidos, se puede concluir que: 1) no existen diferencias entre los riesgos psicosociales presente en los trabajadores que ya se encuentran calificados por enfermedad de origen laboral y los trabajadores que se encuentran a la espera de calificación por 
enfermedad laboral, dando validez a la hipótesis nula, 2) la empresa no está implementando o implementando de manera inadecuada un programa de intervención respecto a los factores de riesgos laborales, programa que debe estar liderado por la alta dirección de la empresa, para que se demuestre el compromiso hacia los trabajadores en lo que se refiere a mantener espacios de trabajo seguros y saludables, sin importar las condiciones particulares de todos los miembros colaboradores de la organización; 3) el trabajador también tiene la responsabilidad de contribuir con la implementación de las medidas tanto correctivas como preventivas en materia de SST, las cuales permiten identificar de manera temprana las condiciones inseguras para su salud, así como los mecanismos que permiten minimizar los riesgos con miras a disminuir la incidencia y prevalencia de casos de enfermedad laboral en la empresa y en este caso particular a los efectos que dichas condiciones inseguras puedan generar a nivel de riesgo psicosocial; 4) las organizaciones deben estar más abiertas al desarrollo de este tipo de actividades, ya que, de manera objetiva, permite evidenciar las falencias que el SG-SST esté presentando en la actualidad, debido a situaciones de confort que se generan por la cotidianidad de la actividad laboral, tanto para los trabajadores como para los jefes de área, coordinadores de SST y alta gerencia; 5) todo especialista en SST debe conocer los mecanismos de aplicación de la batería de factores psicosociales con la finalidad no solo de recibir la documentación referente a los resultados de aplicación, sino que esté capacitado para entender el planteamiento que el profesional estable en dicho documento, respecto al manejo que se le debe dar a dicha información a nivel organizacional, con la finalidad de que los aspectos que deben mejorarse puedan cumplirse en los tiempos establecidos, a través de un trabajo interdisciplinario y 6) importancia de establecer climas laborales que favorecen el desarrollo a nivel personal, social y psíquico de los empleados, proyectados en un futuro tanto personal como organizacional, donde sus servicios sean vistos no desde un rango de empleados simplemente, sino asumiendo el plus de colaboradores.

\section{Referencias}

Andrade, F., Alejo, O. \& Armendariz, C. (2018). Método inductivo y su refutación deductista. Conrado. Revista Pedagógica de la Universidad de Cienfuegos, 14 (63), 117-122. Recuperado de http://scielo.sld.cu/pdf/rc/v14n63/1990-8644-rc-14-63-117.pdf

Ansoleaga, E. (2015). Indicadores de salud mental asociados a riesgo psicosocial laboral en un hospital público. Revista Médica de Chile, 143 (1), 47-55. Recuperado de https://scielo.conicyt.cl/scielo.php?script=sci_arttext\&pid=S0034-98872015000100006

Arias, F. (2012). El proyecto de investigación. Introducción a la metodología científica (6. ${ }^{a}$ ed.). Caracas: Editorial Episteme.

Arias, P., Carvajal, R., \& Cruz, Á. (2012). Ausentismo en trabajadores con reintegro laboral. Revista Colombiana De Salud Ocupacional, 2 (4), 14-18. Recuperado de https://revistas.unilibre.edu.co/index.php/rc_salud_ocupa/article/view/4845/4133

Cacua, F., Carvajal, H., \& Hernández, N. (2017). Condiciones de trabajo y su repercusión en la salud de los trabajadores de la plaza de mercado la nueva sexta, Cúcuta. Psicoespacios, 11 (19), 99-119. Recuperado de https://dialnet.unirioja.es/servlet/articulo?codigo=6109872 


\section{Riesgos psicosociales en trabajadores calificados por enfermedad laboral frente a trabajadores que esperan por calificación}

Congreso de Colombia (2002). Ley 776. Organización, administración y prestaciones del Sistema General de Riesgos Profesionales. Bogotá, Colombia. Diario Oficial $\mathrm{N}^{\circ} 45037$. Recuperado de https://barranquilla.eregulations.org/media/Ley\%20776\%20de\%202002\%20Sistema\%20General\%20de\%20Ries gos\%20Profesionales.pdf).

Delgado, D., \& Aguilera, A. (2017). Vivencias de la reubicación laboral, en trabajadores mineros con silicosis de la división Andina-Codelco, Chile, 2010. Asociación De Especialistas En Prevención Y Salud Laboral, 1-26. Recuperado de https://www.aepsal.com/wp-content/uploads/2017/01/EstudiocompletoSilicosisChile.pdf

Flores, E., Miranda, M. \& Villasís, M. (2017). El protocolo de investigación VI: cómo elegir la prueba estadística adecuada. Estadística inferencial. Revista Alergia México, 64 (3), 364-370. Recuperado de http://www.scielo.org.mx/pdf/ram/v64n3/2448-9190-ram-64-03-0364.pdf

Garzón, L. (2019). Proceso de reintegro y reubicación laboral, una mirada en el ámbito colombiano (Profesional en gestión de la seguridad y salud laboral). Politécnico Gran Colombiano.

Gómez, F. (2019). Modelo de liderazgo basado en la motivación y adaptabilidad, en el proceso de recepción de colaboradores reubicados por condiciones de salud, Avianca, seccional Cali (Maestría en Gestión Social Empresarial). Universidad Externado de Colombia.

Gómez, M., \& Calderón, P. (2017). Salud mental en el trabajo: entre el sufrimiento en el trabajo y la organización saludable. Katharsis, 23, 177-201. Recuperado de file://D:/Descargas/Dialnet-SaludMentalEnElTrabajo5888108.pdf

Gómez, V., Segura, S., Castrillón, D., \& Perilla, L. (2016). Estandarización de una batería para la evaluación de factores de riesgo psicosociales laborales en trabajadores colombianos. Acta Colombiana De Psicología, 19(2), 221-238. Recuperado de http://www.scielo.org.co/pdf/acp/v19n2/es_v19n2a10.pdf

González, R. (2016). ¿es la visión integral actual del ser humano, suficiente? Medisur, 14 (6), 816-821. Recuperado de http://scielo.sld.cu/scielo.php?script=sci_arttext\&pid=S1727-897X2016000600022

Gonzales, E. (2017). Factores del riesgo psicosocial y el estrés laboral en los colaboradores de una empresa de servicios generales del Alto Talara (Profesional de Psicología). Universidad César Vallejo.

Hernández, R., Fernández, C., \& Baptista, P. (2014). Metodología de la investigación (6. ${ }^{a}$ ed.) Ciudad de México: McGraw-Hill / Interamericana Editores.

Herrán-Villalba, V., Lugo-Calderón, E., Puello-Martínez, A., \& Ruiz-Restrepo, A. (2016). Nivel de conocimiento en riesgos laborales: el caso de trabajadores de servicios generales en una universidad. IPSA Scientia, Revista científica Multidisciplinaria, 1(1), 43-51. Recuperado a partir de https://latinjournal.org/index.php/ipsa/article/view/893

Ministerio de la Protección Social. (2008). Resolución 2646. Responsabilidades para la identificación, evaluación, prevención, intervención y monitoreo permanente de la exposición a factores de riesgo psicosocial en el trabajo y determinación del origen de las patologías causadas por el estrés ocupacional. Bogotá, Colombia. Diario Oficial $\mathrm{N}^{\circ} \quad 47059 . \quad$ Recuperado

de https://www.icbf.gov.co/cargues/avance/docs/resolucion_minproteccion_2646_2008.htm

Ministerio del Trabajo. (2014). Decreto 1477. Tabla de enfermedades laborales. Bogotá, Colombia. Diario Oficial N 49.234. Recuperado de https://www.icbf.gov.co/cargues/avance/docs/decreto_1477_2014.htm

IPSA SCIENTIA - Vol. 5 N$^{\circ} 1-2020$ 


\section{Riesgos psicosociales en trabajadores calificados por enfermedad laboral frente a trabajadores que esperan por calificación}

Ministerio del Trabajo. (2019). Resolución 2404. Batería de Instrumentos para la evaluación de Factores de Riesgo Psicosocial, Guía Técnica General para la Promoción, Prevención e Intervención de los Factores Psicosociales y sus efectos en la población trabajadora y sus protocolos específicos. Bogotá, Colombia. Diario Oficial ํㅜ 51023. Recuperado de https://www.icbf.gov.co/cargues/avance/docs/resolucion_mtra_2404_2019.htm

Moreno, A. (2018). El razonamiento implicado en el estudio del muestreo. Aportes para la enseñanza de la estadística inferencial. Acta Latinoamericana de Matemática Educativa, 31 (1), 757-763. Recuperado de http://funes.uniandes.edu.co/13552/1/Moreno2018El.pdf

Moreno, L., Vaca, S., Martínez, D., Suasnavas, P., Cárdenas, I., \& Gómez, A. (2018). Diseño y validación de un cuestionario para el diagnóstico de riesgos psicosociales en empresas ecuatorianas. Ciencia \& Trabajo, 20 (63), 160-168. Recuperado de https://scielo.conicyt.cl/scielo.php?script=sci_arttext\&pid=S0718-24492018000300160

Muñoz-Rojas, D., Vargas-Ortiz, L., \& Latta-Arias, C. (2018). Estructura del clima organizacional en algunas universidades públicas del norte de Colombia. IPSA Scientia, Revista científica Multidisciplinaria, 3(1), 17-22. https://doi.org/10.25214/27114406.921

Organización Internacional del Trabajo. Equipo Técnico de Trabajo Decente de la OIT para América Central, Haití, Panamá y República Dominicana. (2020). La organización del trabajo y los riesgos psicosociales: una mirada de género. $\quad$ Disponible

en http://www.saltra.una.ac.cr/images/SALTRA/Documentacion/Publicaciones_OIT/Anexo_OIT_3.pdf

Otárola, M. (2015). Prevalencia de la ansiedad y de la depresión en una muestra del personal de servicios generales de la Universidad de Costa Rica, que laboran en las secciones de transportes, seguridad y tránsito, Sede Rodrigo Facio (Licenciatura en Psicología). Universidad de Costa Rica.

Ramos, C. (2019). Problema, objetivos y análisis de datos: una reflexión metodológica en la investigación psicológica. Avances en Psicología, 17 (1), 9-19. Recuperado de file://D:/Descargas/PROBLEMAOBJETIVOSYANLISISDEDATOSUNAREFLEXINMETODOLGICAENLA INVESTIGACINPSICOLGICA.pdf

Riaño, M. (2017). Economía de la salud y seguridad en el trabajo: un análisis de costos y beneficios desde las perspectivas del asegurador y de la empresa (Doctorado en Ciencias Económicas). Universidad Nacional de Colombia.

Rubio, N., \& Luna, J. (2014). Análisis del desempeño de la batería de evaluación de factores psicosociales en Colombia. Revista Salud Pública, $17 \quad$ (1), 33-46. Recuperado de https://www.scielosp.org/article/rsap/2015.v17n1/33-46/

Ruiz, A. \& López, J. (2020). Cómo mejorar la comunicación de estadísticos inferenciales en ciencias de la salud. Revista Española de Comunicación en Salud, 11 (1), 139-145. Recuperado de file:///D:/Descargas/5173-9325-1PB.pdf

Torres, M., \& Torres, N. (2017). Trabajo y salud: la Corte Constitucional frente a la tutela por accidentes y enfermedades laborales. Revista De Salud Pública, 19 (6), 772-779. Recuperado de https://scielosp.org/pdf/rsap/2017.v19n6/772-779/es

UGT Andalucía (2009). Guía de la prevención de riesgos psicosociales en el trabajo. Andalucía: Lienzo Gráfico.

IPSA SCIENTIA - Vol. 5 No $1-2020$ 